\title{
Efecto de dos campos magnéticos sobre el crecimiento micelial y propiedades sensoriales del hongo Pleurotus ostreatus
}

\section{Effect of two magnetic fields on the mycelial growth and sensorial properties of the mushroom Pleurotus ostreatus}
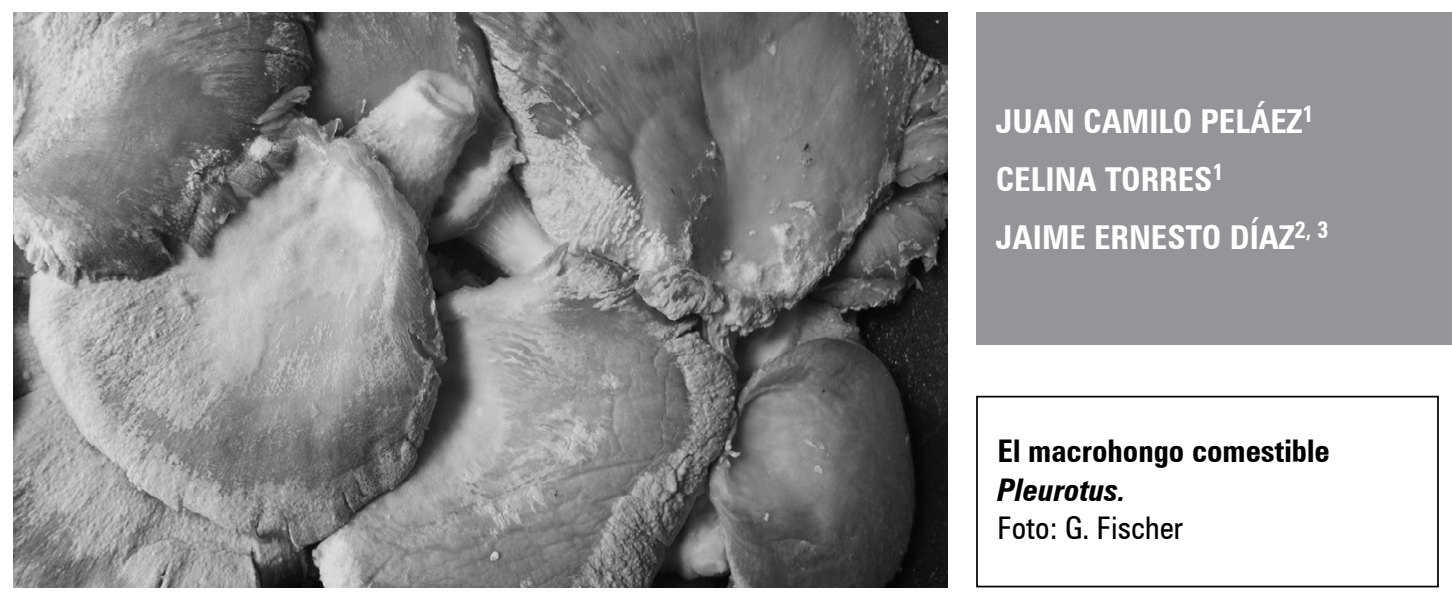

\section{RESUMEN}

Se evaluó la acción de los campos magnéticos estáticos sobre el crecimiento micelial del hongo comestible pleuroto Pleurotus ostreatus ([Jacq: Fries] Kummer), cepa WC-923, valorado por sus propiedades medicinales y nutritivas. Los campos magnéticos se produjeron empleando imanes de ferrita con intensidades de $125 \mathrm{mT}$ y $250 \mathrm{mT}$. Los periodos de exposición fueron 10, 30, 60 minutos, 48 horas y 7 días. Los datos de crecimiento micelial se tomaron el día 7 y se observó que el tratamiento compuesto por el imán de $250 \mathrm{mT}$ y el periodo de exposición 7 días presentó el mayor diámetro con $8,8 \mathrm{~cm}$ del micelio, indicando además que dicho tratamiento presentó la variación más baja con relación a los demás. En la prueba de Dunnett, el tratamiento de $125 \mathrm{mT}$ y 7 días de exposición fue el único que arrojó diferencias significativas frente al grupo control. Los tratamientos $125 \mathrm{mT}$ y $250 \mathrm{mT}$, con 7 días de exposición, mostraron cambios en las características aromáticas al presentarse olores anisados en los primeros 10 días que posteriormente se convirtieron en almendrados. La observación física mostró que algunos micelios formaban anillos centrales en alto relieve los cuales secretaban un metabolito anaranjado que se caracteriza como nematicida. Se concluye que la aplicación de campos magnéticos incide en el crecimiento positivo del hongo.

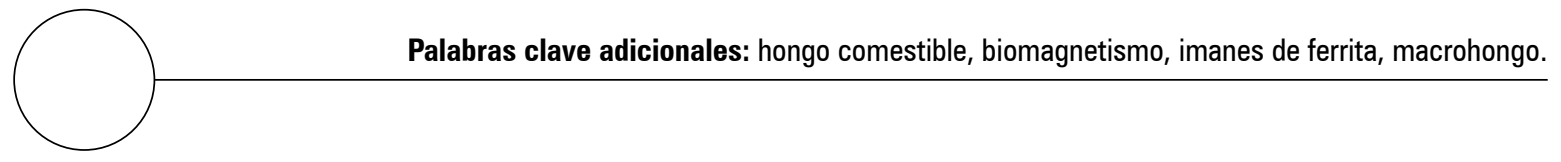

Facultad de Ciencias Naturales y Exactas, Departamento de Biología, Programa de Biología, Universidad del Valle, Cali (Colombia).

2 Facultad de Ingeniería, Escuela de Ingeniería de Recursos Naturales, Programa de Ingeniería Agrícola, Universidad del Valle, Cali (Colombia).

3 Autor para correspondencia. jaime.diaz@correounivalle.edu.co 


\section{ABSTRACT}

The present study evaluated the action of static magnetic fields on the mycelial growth of the edible oyster mushroom Pleurotus ostreatus ([Jacq: Fries)] Kummer) strain WC-923, known for its high medicinal and nutritional values. Magnetic fields were produced using ferrite magnets with intensities of $125 \mathrm{mT}$ and $250 \mathrm{mT}$. The exposure periods were 10, 30, and 60 minutes, 48 hours and 7 days. Mycelial growth data were taken on day 7 and it was observed that the magnet treatment of $250 \mathrm{mT}$ with the exposure period of 7 days showed the greatest mycelial diameter $(8.8 \mathrm{~cm})$, with indication that this treatment showed the lowest variation compared to the others. In the Dunnett post-Anova test, the treatment of $125 \mathrm{mT}$ with 7 days of exposure was the only one that showed significant differences compared with the control group. The treatments of $125 \mathrm{mT}$ and $250 \mathrm{mT}$ with 7 days of exposure showed changes in the aromatic characteristics with aniseed odors during the first 10 days, which later became almond-like. Physical observation showed that some mycelia built central rings in high relief, which secreted an orange colored metabolite characterized as nematicide. It was concluded that the application of magnetic fields has a positive growth effect on the fungus.

Additional key words: edible mushroom, biomagnetism, ferrite magnets, macrofungus.

Fecha de recepción: 10-04-2013

Aprobado para publicación: 28-05-2013

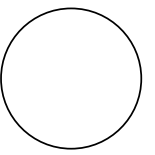

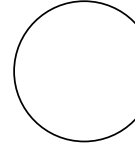

El pleuroto (Pleurotus ostreatus [Jacq: Fries] Kummer), igual que otros macrohongos, tiene necesidades nutricionales como el carbono, nitrógeno, azufre, fósforo, potasio, magnesio (Chang y Miles, 2004; Herrera, 2008) y cantidades menores de otros elemento como calcio, hierro, zinc, manganeso, cobre, molibdeno y níquel (Walker y White, 2005). Los factores abióticos y bióticos deben ser suministrados y controlados para un óptimo desempeño del organismo (Stamets y Chilton, 1983; Wainwright y Liras, 1995), sin embargo, existe un factor más que afecta a todos los seres vivientes por igual dado que la vida sobre la tierra se halla inmersa en un campo geomagnético (Kato y Shigemitsu, 2006), dicho factor conocido como campo magnético es intangible pero igual de importante a los demás y puede alterar funciones en procesos enzimáticos, celulares fisiológicos y reproductivos (Adey, 1993; Galland y Pazur, 2005; Goldsworthy et al., 1999; Nair, 1989).
En Colombia, no existen experiencias en este tipo de cultivos, por lo que se hace necesario realizar investigaciones que permitan generar el conocimiento para alcanzar un proceso a nivel industrial y obtener un compuesto o producto derivado del micelio de los macro-hongos. Por tanto, este estudio se realizó para evaluar en condiciones de laboratorio los efectos biológicos de campos magnéticos estáticos sobre el hongo P. ostreatus determinando las intensidades magnéticas más favorables para el desarrollo micelial y sus propiedades sensoriales.

\section{MATERIALES Y MÉTODOS}

La cepa empleada Pleurotus ostreatus ([Jacq: Fries] Kummer) WC-923, fue facilitada por la planta de Bioprocesos de la Universidad de Caldas y corresponde a una segunda generación. La cepa fue replicada inoculando trozos de $5 \mathrm{~mm}^{2}$ de la 
cepa madre, con asas de inoculación estériles, en cinco cajas de Petri estándar con medio de cultivo agar PDA (papa-dextrosa-agar), las cajas se incubaron a $28^{\circ} \mathrm{C}$ durante $8 \mathrm{~d}$ hasta observar que el cultivo había invadido completamente el medio y posteriormente se conservaron mediante refrigeración a $-10^{\circ} \mathrm{C}$. Este proceso se realizó en cuarto ambiental de la sección de botánica y los laboratorios de Microbiología y Fitosanidad de la Universidad del Valle, sede Meléndez, donde la temperatura fue de $28 \pm 1^{\circ} \mathrm{C}$ y la humedad relativa de $75 \pm 2 \%$. La etapa de exposición del hongo a campos magnéticos se realizó en el Laboratorio de Aguas y Suelos Agrícolas (LASA) de la Universidad del Valle, sede Meléndez, en Cali.

Una vez replicado el micelio de la cepa madre de $P$. ostreatus se procedió a inocular con $5 \mathrm{~mm}$ del micelio de estas cajas, cada uno de los tratamientos del experimento, y se estableció el grupo control. En la base de cada caja se colocaron imanes toroide de ferrita de $125 \mathrm{mT}$ (diámetro exterior $5,8 \mathrm{~cm}$ e interior de $2,4 \mathrm{~cm}$ ), y de 250 $\mathrm{mT}$ (diámetro exterior $7,8 \mathrm{~cm}$ e interior de 3,1 $\mathrm{cm})$. Los imanes de carácter industrial fueron previamente calibrados en el Laboratorio de Física Ambiental de la Universidad del Valle, empleando una sonda electromagnética. Las cajas de Petri fueron expuestas al campo magnético de imanes colocados en la parte inferior de ellas durante periodos de 10, 30, $60 \mathrm{~min}, 48 \mathrm{~h}$ y $7 \mathrm{~d}$.

Al finalizar cada ensayo se realizaron las mediciones del crecimiento micelial, empleando un pie de rey para determinar el diámetro. El reconocimiento de las características aromáticas se estimó al percibir cambios drásticos en su olor y por su aspecto visual. Macroscópicamente se observaron todas las características como textura, color y metabolitos exudados y se identificaron con el microscopio, hifas y fíbulas a las que se les midieron sus diámetros mediante el software instalado en la cámara de un microscopio óptico compuesto de marca Coleman (Coleman Equipamentos para Laboratório Com., Santo André
[SP], Brasil) con aumento de 1-1000x y ocular EW P10x/20mm.

El experimento se desarrolló mediante un arreglo de dos factores (intensidad magnética y tiempo de exposición, $2 \times 5$ ), con 10 tratamientos: dos intensidades magnéticas de $125 \mathrm{mT}$ y $250 \mathrm{mT}$ y cinco tiempos de exposición de 10, 30, $60 \mathrm{~min}, 48 \mathrm{~h}$ y $7 \mathrm{~d}$. Se realizaron cinco repeticiones por tratamiento. Para las repeticiones se emplearon cajas de Petri con medio de cultivo y el hongo inoculado. El control consistió de otras cinco cajas con medio de cultivo, a las que no se les aplicó magnetismo. Se realizaron cinco ensayos, cada uno con un total de $55 \mathrm{ca}$ jas de Petri inoculadas con micelio de pleuroto. Posteriormente se realizó un análisis de varianza dos vías y una prueba pos Anova de Tukey, luego se desarrolló un modelo completamente al azar para realizar una comparación entre el control y los demás tratamientos, empleando el test de Dunnet.

\section{RESULTADOS Y DISCUSIÓN}

\section{Crecimiento miceliar}

Los crecimientos miceliares con mayor diámetro se presentan para los tratamientos $125 \mathrm{mT}$ y 250 $\mathrm{mT}$ con un periodo de exposición de $7 \mathrm{~d}$; los valores del diámetro de $5,84 \mathrm{~cm}$ y $8,82 \mathrm{~cm}$ respectivamente, fueron superiores al del control (5,44 $\mathrm{cm})$ y a los demás tratamientos con tiempos de exposición inferiores. Sin embargo, tratamientos con tiempos de exposición de 60 min para ambas intensidades y de $30 \mathrm{~min}$ para $125 \mathrm{mT}$, igualmente, mostraron buen crecimiento pero no superior al control (figura 1).

El análisis de Anova mostró que no existen diferencias significativas en la intensidad del campo, pero sí en el factor tiempo, donde el periodo de exposición de 7 d mostró diferencias significativas con un nivel de confianza del 99\% (figura 2). 


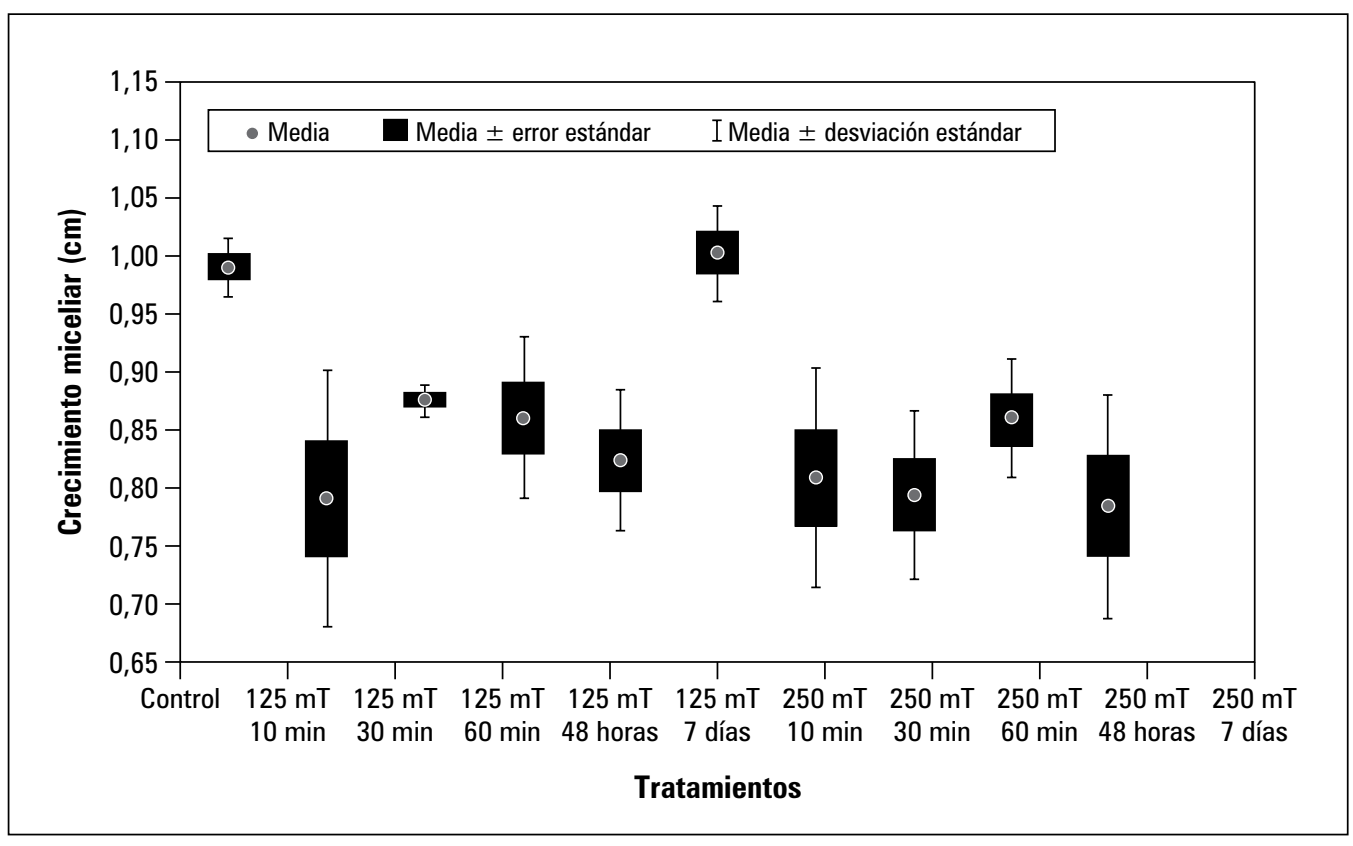

Figura 1. Efecto de los campos magnéticos 125 y $250 \mathrm{mT}$ sobre el crecimiento micelial de Pleurotus ostreatus, registrado en cinco diferentes tiempos.

La prueba de Anova para la interacción entre los factores reveló que las diferencias significativas se encuentran en los niveles de $7 \mathrm{~d}$ para las dos intensidades, sin embargo se encontró que estos dos niveles no son estadísticamente diferentes entre sí. La prueba de Dunnett mostró que el tratamiento $250 \mathrm{mT}$ durante $7 \mathrm{~d}$ con un $P=0,026887$ tuvo un promedio significativamente mayor que el del control y con poco variación (figura 1).

Los resultados muestran consistencia con los encontrados por Javanmardi et al. (2008), quienes determinaron que $P$. ostreatus responde de manera positiva y negativa en el crecimiento micelial a intensidades magnéticas entre 6 y $12 \mathrm{mT}$.

La manera como el pleuroto parece responder a los campos magnéticos, se puede explicar por la existencia de dos mecanismos de magnetorrecepción muy conocidos en plantas y hongos filamentosos; el primero es la sensibilidad de estos debido a la presencia de fotorreceptores de luz (criptocromos) y el segundo a la existencia de mecanismo de resonancia iónica ciclotrónica (ICR) (Galland y Pazur, 2005).

Los criptocromos fúngicos presentes en los grupos ascomycetes, basidiomycetes y zygomycetes son relacionados con la reparación del ADN dañado por luz ultravioleta (Corrochano, 2011). Este fotorreceptor posee una enzima fotoliasa (FAD) unida a un pigmento antena (MTHF) (Heintzen, 2012), el cual en presencia de luz azul, forma dos subproductos derivados de la enzima FDA y el triptofanil, denominados pares radicales, que pueden estar en estado singlete $y / 0$ triplete, que en presencia de campos magnéticos estáticos débiles, parecen mejorar las tasas de formación de dichos pares radicales prolongando la vida media de estos dando como resultado un cambio a nivel fisiológico debido al incremento de la señalización celular y el rendimiento de las reacciones (Ahmad et al., 2007), lo cual explicaría la formación de metabolito secundario color naranja en la mayoría de los tratamientos con el campo magnético de $125 \mathrm{mT}$ ya que es el más débil. 


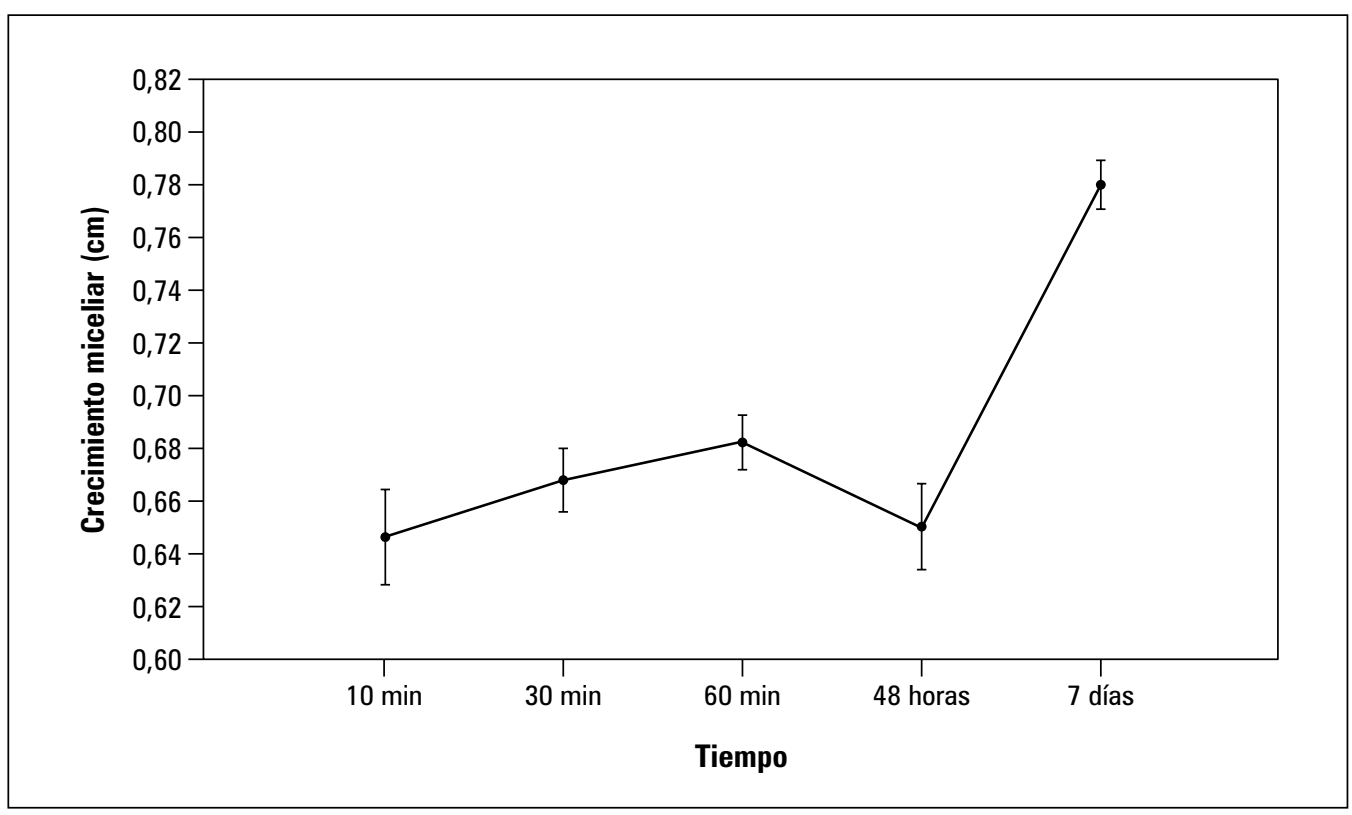

Figura 2. Efecto del tiempo de exposición de los campos magnéticos de 125 y $250 \mathrm{mT}$ sobre el crecimiento micelial de Pleurotus ostreatus. Las barras sobre los promedios indican desviación estándar.

Por otra parte el modelo de resonancia iónica ciclotrónica predice que los iones de calcio se moverán circularmente por las líneas de los campos geomagnéticos a través de los canales de calcio. Sin embargo, si se le superpone un campo electromagnético con una frecuencia definida, los iones cambiarán su ángulo de movimiento y se trasladarán helicoidalmente, proceso que alteraría la velocidad de entrada de los iones y transferiría energía cinética a los canales, dando como resultado un flujo más acelerado en las membranas y una mayor entrega de energía hacia otros compartimentos de la célula (Liboff, 1985). Lo anterior podría explicar la eficiencia del crecimiento micelial obtenida en el tratamiento 250 mT y 7 d de exposición.

\section{Propiedades sensoriales: olores característicos}

En la tabla 2 se presenta la variación del olor de los micelios antes y después de $10 \mathrm{~d}$ de exposición a los tratamientos. Se observó que los tratamientos con imanes de $125 \mathrm{mT}$ y $250 \mathrm{mT}$ durante $7 \mathrm{~d}$ presentaron olores a almendra muy tenues pero fuertes de, anís mientras que los de- más sí poseían dicha fragancia de almendra pero no anisada lo que es habitual presentándose hasta la fructificación del hongo (Stamets, 2000). Por otra parte, después del décimo día, estos tratamientos ya no tenían ese olor fuerte de anís pero si de almendra muy tenue como si hubiesen producido una inversión de olores entre estados maduros e inmaduros.

De acuerdo con Beltrán-García et al. (1997), los aromas que se encuentran en mayor cantidad en $P$. ostreatus corresponden a: 3-octanona, 3-octanol, 1-octen-3-ol, benzaldehído, 1-octanol y ácido benzoico. Dichos compuestos tienen actividad antimicrobial, pero también otorgan al hongo ciertos olores característicos como cacao amargo, anís, almendra, levadura y afrutado (Gallois et al., 1990; Schindler y Schmid, 1982). Es probable que la exposición a campos magnéticos con tiempo prolongado afecte la producción de compuestos volátiles.

Con relación a los aromas percibidos en los micelios antes y después del décimo día, se observó que aunque no todos los micelios presentaban el 
Tabla 2. Olores característicos de los micelios según el tratamiento.

\begin{tabular}{|l|l|l|l|l|}
\hline \multirow{2}{*}{ Tratamientos } & \multicolumn{2}{c|}{ Antes del día 10} & Almendra & Anisado \\
\cline { 2 - 5 } & Almendra & Anisado & casi nulo & fuerte \\
\hline $125 \mathrm{mT} / 10 \mathrm{~min}$ & fragante & sin aroma & casi nulo & fuerte \\
\hline $125 \mathrm{mT} / 30 \mathrm{~min}$ & fragante & sin aroma & casi nulo & fuerte \\
\hline $125 \mathrm{mT} / 60 \mathrm{~min}$ & fragante & sin aroma & casi nulo & fuerte \\
\hline $125 \mathrm{mT} / 48 \mathrm{~h}$ & fragante & sin aroma & casi nulo & fuerte \\
\hline $125 \mathrm{mT} / 7 \mathrm{~d}$ & fragante & sin aroma & fragante & sin aroma \\
\hline $250 \mathrm{mT} / 10 \mathrm{~min}$ & casi nulo & fuerte & casi nulo & fuerte \\
\hline $250 \mathrm{mT} / 30 \mathrm{~min}$ & fragante & sin aroma & casi nulo & fuerte \\
\hline $250 \mathrm{mT} / 60 \mathrm{~min}$ & fragante & sin aroma & casi nulo & fuerte \\
\hline $250 \mathrm{mT} / 48 \mathrm{~h}$ & fragante & sin aroma & casi nulo & fuerte \\
\hline $250 \mathrm{mT} / 7 \mathrm{~d}$ & fragante & sin aroma & fragante & sin aroma \\
\hline
\end{tabular}

mismo olor e intensidad para los tratamientos $125 \mathrm{mT}$ y $250 \mathrm{mT}$ con $7 \mathrm{~d}$ de exposición, los olores se modificaron de anisado fuerte a almendra tenue, lo cual es habitual encontrar hasta la fructificación del hongo (Stamets, 2000).

Según Jong and Birmingham (1993), los olores son compuestos volátiles de los alimentos cuyas propiedades aromáticas son percibidas por la nariz. Cuando estos aromas están ausentes en el hongo, indica que está en estado inmaduro o demasiado maduro. Los compuestos volátiles en mayor cantidad encontrados en $P$. ostreatus son 3-octanona, 3-octanol, 1-octen-3-ol, benzaldehído, 1-octanol y ácido benzoico. Dichos compuestos tienen actividad antimicrobial (Beltrán-García et al., 1997) pero también otorgan al hongo ciertos olores característicos como cacao amargo, anís, almendra, levadura y afrutado (Badcock, 1939; Gallois et al., 1990; Maga, 1976; Schindler and Schmid, 1982). Se puede pensar que la acción de los campos magnéticos con tiempo de exposición prolongada, afectan la producción de compuestos volátiles debido a la alteración de las reacciones que los producen en los diferentes estados de crecimiento.

\section{Características físicas, químicas y morfológi- cas de los micelios resultantes}

Algunos micelios presentaban un anillo en el centro en alto relieve y a su vez gotas anaranjadas de un exudado (metabolito secundario) y en ocasiones casi transparente. Otros micelios eran planos y no presentaban este exudado. Este compuesto orgánico fue analizado mediante cromatografía de gases en los laboratorios de química de la Universidad del Valle, determinando entre sus componentes ácido acético el cual tiene propiedades nema-tóxicas. Como señalaron Truong et al. (2007), este tipo de compuestos solo se acumulan en toxoquistes, que son esféricas y crecen solo en hifas aéreas en las que la división celular ha terminado tras la maduración del hongo. Otros autores encontraron la acumulación de ácido linoléico (Satou et al., 2008) y ácido trans-2-decanedioico (Kwok et al., 1992).

Lo anterior permite sugerir que la formación de dicho anillo en el centro de los micelios y la secreción del metabolito anaranjado en los bordes de este, puede estar relacionada con el estrés ge- 
nerado por el tratamiento $250 \mathrm{mT}$ con $7 \mathrm{~d}$ de estimulación, que posibilitó después de ese periodo, la formación de estructuras especializadas para la defensa contra nematodos, siguiendo la inclinación de las líneas del flujo magnético por parte del micelio aéreo que ya ha dejado de dividirse.

Por otra parte, los resultados presentaron similitudes con reportes de Iwalokun et al. (2007) quienes indicaron que los extractos orgánicos de $P$. ostreatus muestran actividad sobre bacterias Gram positivas y Gram negativas, señalando a terpenoides y compuestos fenólicos como los responsables del efecto antibacteriano, lo cual solo se produce en micelios maduros-viejos o fructificados (Nordbring-Hertz et al., 2006; Stamets, 2000).

En los análisis de las características macroscópicas de $P$. ostreatus se observó que existía una gran variación en las morfologías de los micelios y una pequeña producción de metabolito antinemátodos por parte de algunos tratamientos. Según Pazur et al. (2007) existe una posible explicación para esta morfología en el crecimiento, basada en el concepto de la magnetotaxia, que indica que organismos como algunas bacterias orientan su locomoción en ambientes acuíferos hacia las líneas del flujo del campo magnético para encontrar fácilmente un ambiente favorable y rico en elementos como oxígeno u otro compuesto indispensable. Lo anterior podría indicar que el hongo creció siguiendo las líneas invisibles que generaba el campo magnético para facilitar la búsqueda de los nutrientes que necesitaba para su desarrollo, pero la explicación anterior no aclara por qué la morfología apareció solo después del séptimo día y no durante la primera etapa de crecimiento.

\section{CONCLUSIONES}

El tratamiento de $250 \mathrm{mT}$ y 7 d de exposición mostró la mayor efectividad al aumentar los grosores de las hifas y las fíbulas que son indispensables para el desarrollo y maduración del macrohongo.

Los campos magnéticos acompañados de periodos de exposición específicos, pueden producir cambios a nivel fisiológico y morfológico del hongo $P$. ostreatus como la producción de un metabolito secundario y aromas derivados de compuestos volátiles en etapas tempranas de desarrollo.

Debido a que existen diversos mecanismos de respuesta de los seres vivos a los efectos de los campos magnéticos, probablemente las respuestas del pleuroto hayan sido producto de una sinergia entre dichos mecanismos o simplemente cada mecanismo hizo efecto a determinado tratamiento.

Se sugiere para posteriores ensayos analizar dichos compuestos con técnicas avanzadas que permitan identificar compuestos que puedan tener aplicación en la industria de los alimentos y perfumería. 


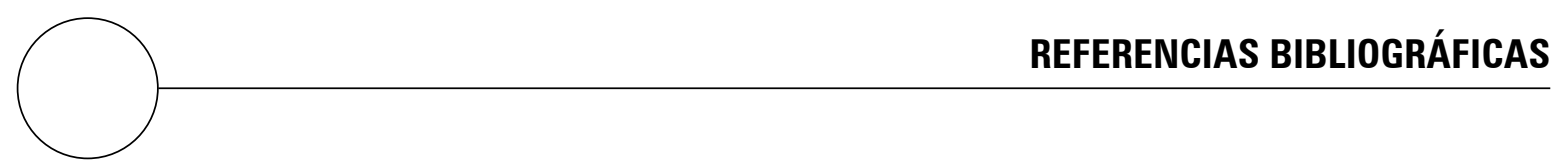

Adey, W.R. 1993. Biological effects of electromagnetic fields. J. Cell. Biochem. 51, 410-416.

Ahmad, M., P. Galland, T. Ritz, R. Wiltschko y W. Wiltschko. 2007. Magnetic intensity a Vects cryptochrome-dependent responses in Arabidopsis thaliana. Planta Médica 225, 615-624

Badcock, E.C.1939. Preliminary account of the odour of wood-destroying fungi in culture. Trans British Mycol. Soc. 23(2), 188-198.

Beltrán-García, M. J., M. Estarron-Espinosa y T. Ogura. 1997. Volatile compounds secreted by the oyster mushroom (Pleurotus ostreatus) and their antibacterial activities. J. Agr. Food Chem. 45(10), 4049 4052 .

Chang, S.T.y P.G. Miles. 2004. Mushrooms: Cultivation, nutritional value, medicinal effect, and environmental impact. $2^{\text {nd }}$ ed. CRC Press, Boca Raton, FL.

Corrochano, L.M. 2011. Fungal photobiology: a synopsis. IMA Fungus 2(1), 25-28.

Galland, P. y A. Pazur. 2005. Magnetoreception in plants. J. Plant Res. 118(6), 371-389.

Gallois, A., B. Gross, D. Langlois, H.E. Spinnler y P. Brunerie. 1990. Influence of culture conditions on production of flavour compounds by 29 ligninolytic Basidiomycetes. Mycol. Res. 94(4), 494-504.

Goldsworthy, A., H. Whitney y E. Morris. 1999. Biological effects of physically conditioned water. Water Res. 33(7),1618-1626.

Heintzen, C. 2012. Plant and fungal photopigments. Wiley Interdisciplinary Reviews: Membrane Transport and Signaling 1(4), 411-432.

Herrera, J.R. 2008. Viaje al asombroso mundo de los hongos. Consejo Nacional de Ciencia y Tecnología, Fondo de Cultura Economica, México.

Iwalokun, B.A, U.A. Usen, A.A. Otunba y D.K. Olukoya. 2007. Comparative phytochemical evaluation, antimicrobial and antioxidant properties of Pleurotus ostreatus. Afr. J. Biotechnol. 6 (15), 1732-1739.

Javanmardi, J., M. Ranjbar y G. Shams. 2008. Effect of a magnetic field on growth indices of oyster mushroom (Pleurotus florida). Int. Soc. Mushrooms Sci. 17(35), 459-466.

Jong, S.C. y J.M. Birmingham. 1993. Medicinal and therapeutic value of the Shiitake mushroom. Adv. Appl. Microbiol. 39,153-184.

Kato, M. y T. Shigemitsu. 2006. Electromagnetics in biology. Springer, Tokyo.

Kwok, O.C.H., R. Plattner, D. Weisleder y D. T. Wicklow. 1992. A nematicidal toxin from Pleurotus ostreatus NRRL 3526. J. Chem. Ecol. 18(2), 127-136.

Liboff, A.R. 1985. Geomagnetic cyclotron resonance in living cells. J. Biol. Physics 13(4), 99-102.

Maga, J.A. 1976. The potential of certain fungi as sources for natural flavor compounds. Chem. Senses Flavor 2(2), 255-262.

Nair, I. 1989. Biological effects of power frequency electric and magnetic fields. Carnegie Mellon University, Pittsburgh, PA.

Nordbring-Hertz, B., H.-B. Jansson y A. Tunlid. 2006. Nematophagous fungi. pp. 1-11. En: Encyclopedia of Life Sciences. John Wiley \& Sons, Chichester, UK.

Pazur, A., C. Schimek y P. Galland. 2007. Magnetoreception in microorganisms and fungi. Central Eur. J. Biol. 2(4), 597-659.

Satou, T., K. Kaneko, W. Li y K. Koike. 2008. The toxin produced by pleurotus ostreatus reduces the head size of nematodes. Biol. Pharm. Bull. 31(4), 574-576.

Schindler, J. y R.D. Schmid. 1982. Fragrance or aroma chemicals-microbial synthesis and enzymatic transformation-a review. Process Biochem. 17, 2-8.

Stamets, P. 2000. Growing gourmet and medicinal mushrooms. $3^{\text {th }}$ ed. Ten Speed Press, Berkeley, CA. 
Stamets, P. y J.S. Chilton. 1983. The mushroom cultivator: a practical guide to growing mushrooms at home. Agarikon Press, Olympia, WA.

Truong, B.N., K. Okazaki., T. Fukiharu., Y. Takeuchi., K. Futai., X.T. Le y A. Suzuki. 2007. Characterization of the nematocidal toxocyst in Pleurotus subgen. Coremiopleurotus. Mycosci. 48(4), 222-230.
Wainwright, M. y S.M. Liras. 1995. Introducción a la biotecnología de los hongos. Acribia, Zaragoza, España.

Walker, G. y N. White. 2005. Introduction to fungal physiology. pp 1-34. En: Kavanagh, K. (ed.). Fungi: Biology and applications. John Wiley and Sons, Chichester, UK. 